\title{
Update of Immunosenescence in Cerebral Small Vessel Disease
}

\author{
Banghao Jian ${ }^{1 \dagger}$, Mengyan $\mathrm{Hu}^{1 \dagger}$, Wei Cai ${ }^{1,2+}$, Bingjun $\mathrm{Zhang}^{1 *}$ and Zhengqi $\mathrm{Lu}^{1 *}$ \\ ${ }^{1}$ Department of Neurology, Center for Mental and Neurological Disorders and Diseases, The Third Affiliated Hospital of \\ Sun Yat-sen University, Guangzhou, China, ${ }^{2}$ Center of Clinical Immunology, Center for Mental and Neurological Disorders \\ and Diseases, The Third Affiliated Hospital of Sun Yat-sen University, Guangzhou, China
}

Aging of the central nervous system (CNS) is closely associated with chronic sterile lowgrade inflammation in older organisms and related immune response. As an amplifier for neuro-inflammaging, immunosenescence remodels and deteriorates immune systems gradually with the passage of time, and finally contributes to severe outcomes like stroke, dementia and neurodegeneration in elderly adults. Cerebral small vessel disease (CSVD), one of the major causes of vascular dementia, has an intensive connection with the inflammatory response and immunosenescence plays a crucial role in the pathology of this disorder. In this review, we discuss the impact of immunosenescence on the development of CSVD and its underlying mechanism. Furthermore, the clinical practice significance of immunosenescence management and the diagnosis and treatment of CSVD will be also discussed.

Keywords: aging, immunosenescence, cerebral small vessel disease, pathogenesis, inflammaging

\section{INTRODUCTION}

Cerebral small vessel disease (CSVD), a considerable health care problem, contains a wide spectrum of cerebrovascular diseases that primarily affect capillaries, small arteries and small veins in the brain, and brings a serious hazard to aging societies (1). Arteriolosclerosis is the most popular and extensive subtype of CSVD and closely related to the overall health status of the body like aging and hypertension. Thus, such type is also called age-related and vascular risk-factor-related small vessel disease (2). Mounting evidence indicated that arteriolosclerosis is the major cause of ischemic stroke, intracerebral hemorrhage, dementia and raises mortality in elderly people, and the term CSVD in this review is majorly used to discuss arteriolosclerosis (3). The neurobiological basis of the underlying mechanism of CSVD is poorly understood now. However, chronic inflammation, induced by either immune cells or non-immune cells, is closely associated with the aging process of cerebral vessels and related brain metabolism and draws a substantial amount of attention. Therefore, it is of great potential and significance to investigate the role of immune response during aging in the development of CSVD and corresponding cerebral injury.

To better understand the immune system alteration during aging, the term "immunosenescence" raises and represents the deterioration of multiple immune cells and function change of key molecules like cytokines, chemokines and extracellular matrix components (4). It is renowned that immunity acts an emerging role in the pathological process of CSVD in elderly (5). The peripheral immune system, involving lymphocytes, cytokines and antibodies, contributes to the vascular 
disorganization, blood brain barrier (BBB) leakage and immune cells infiltration. Importantly, damaged cells suffer a special stage called senescence-associated secretory phenotype (SASP) from normal states to irreversible aging states. SASP could be found in infiltrated immune cells and residential cells, including endothelial cells, pericytes, neurons and glial cells during CSVD. All the components could form a complex regulatory network and play a crucial role in the pathophysiology of CSVD $(6,7)$. Actually, immunosenescence has a bidirectional impact on multiple disorders development, which often refers to immunosuppression or immune activation. Such pathological process tends to activate an immune response in CSVD and contribute to chronic low-grade inflammation in the CNS, named neuro-inflammaging.

Performing in-depth exploration of the cellular and molecular level of immunosenescence could help physicians and specialists to make a clinical decision and better predict the prognosis of CSVD patients. Through screening key molecules in pathways related to immunosenescence, a regulatory network and vital point target genes could be constructed and found. Specialists could focus on these key modules to build a prognostic model for CSVD patients and provide more precise clinical plan. In addition, drug development could be more time-saving and lower economic toxicity based on these fundamental researches. However, the contribution of immunosenescence in the initiation and progression of CSVD remains obscure and an unmet need for further exploration. This review focuses on the impact of immunosenescence on the development of CSVD and its underlying mechanism, and the meaning of immunosenescence management for CSVD in clinical practice is also discussed.

\section{IMMUNOSENESCENCE IS A PROMISING AMPLIFIER FOR NEURO-INFLAMMAGING}

The concept of immunosenescence states the aging and functional decline throughout the whole immune system. It usually accompanies with a chronic low-grade inflammation status termed inflammaging whose pro-inflammatory mediates remarkably increase (8). This process not only involves the immune system, but tissues such as senescent endothelium, pericytes and adipose cells also. The immune system is composed of innate and adaptive parts, both of which dramatically change in the aging process (9).

\section{Immune System Alterations in Aging Process}

Both adaptive immunity and innate immunity undergo significant changes during the natural process of aging and consequently cause a series of physiological and molecular changes (Table 1). The cell transformation of lymphocytes, along with the antibody and cytokines secretion, represents the function of adaptive immunity while diminished function and phenotype shift towards proinflammatory subtype of macrophages mainly consist of the innate immunity alteration.

The adaptive immune system contains cellular immunity and humoral immunity, composed of $\mathrm{T}$ cells and $\mathrm{B}$ cells in different subpopulations. According to the initial of lymphocytes, T cells origin from bone marrow, and then differentiate and mature in thymus. Later, these functional cells release into circulation and migrate to peripheral immune organs and tissues. Commonly, $\mathrm{T}$ cells can be subdivided into 4 subgroups based on their distinct

TABLE 1 | Summary of immune changes in immunosenescence.

\begin{tabular}{|c|c|c|c|}
\hline Immunity & Cell & Changes & Study \\
\hline \multirow[t]{11}{*}{ Adaptive } & T cell & - TCR pool diminishes & Kilpatrick et al. (10) \\
\hline & & - Subtype changes & Li et al. (11) \\
\hline & & & Jagger et al. (12) \\
\hline & & & Raynor et al. (13) \\
\hline & & - Inflammatory suppression weaken & Fessler et al. (14) \\
\hline & & & Thomas et al. (15) \\
\hline & & - Pro-inflammatory mediates production increase & Hu et al. (16) \\
\hline & & & Singh \& Newman (17) \\
\hline & B cell & - Subtype changes & Bulati et al. (18) \\
\hline & & & Palma et al. (19) \\
\hline & & - Pro-inflammatory mediates production increase & Frasca \& Blomberg (20) \\
\hline \multirow[t]{15}{*}{ Innate } & Monocyte/macrophage & - Chemotaxis/phagocytosis decrease & Antonaci et al. (21) \\
\hline & & & Mahbub et al. (22) \\
\hline & & - Subtype changes & Seidler et al. (23) \\
\hline & & & Sadeghi et al. (24) \\
\hline & & - Pro-inflammatory mediates production increase & Sadeghi et al. (24) \\
\hline & & & Olivieri et al. (25) \\
\hline & & & Jacinto et al. (26) \\
\hline & Dendritic cell & - Number decreases & Della et al. (27) \\
\hline & & - Pro-inflammatory mediates production increase & Splunter et al. (28) \\
\hline & Microglia & - Subtype changes & Yao \& Zhao (29) \\
\hline & & - Pro-inflammatory mediates production increase & Mecca et al. (30) \\
\hline & & & Vida et al. (31) \\
\hline & Neutrophils & - Chemotaxis/phagocytosis decrease & Niwa et al. (32) \\
\hline & NK cell & - Number increase & Gounder et al. (33) \\
\hline & & - Pro-inflammatory mediates production increase & Camous et al. (34) \\
\hline
\end{tabular}


function: helper T cell, cytotoxic T cell, regulatory $\mathrm{T}$ cell (Treg) and memory $\mathrm{T}$ cell. These subtypes participate in pathogen identification, cytokines secretion, immune memory, pathogen killing and immunoregulation, whose function was impaired at varying levels in immunosenescence process. Meanwhile, the cell counts of specific subtypes in functional immune organs including thymic and ratio of different $\mathrm{T}$ cell types, which is widely found, will also change in elderly. For example, the CD4 naïve $\mathrm{T}$ cells continuous decline with the thymic involution while the number of $\mathrm{T}$ cells still remains steady at the periphery via compensatory proliferation, resulting in the loss of diversity of $\mathrm{T}$ cell receptor (TCR) pool $(10,35)$. Besides, both the increase of the $\mathrm{CD} 4 / \mathrm{CD} 8$ ratio and $\mathrm{CD} 8^{+} \mathrm{CD} 28^{-} \mathrm{T}$ cells was observed in elderly people $(11,36)$.

Great attention has been paid to uncover the underlying roles of some subtypes of $\mathrm{T}$ cells as more accurate and precise detecting method develops and clear definition has been made to these cell types. According to the recent studies, the $\mathrm{T}$ cell types that only account for a small part the total population may also make great sense and have tight connection with other cells or stromal components which could be also called microenvironment. It should be noted that the investigation on Tregs, a newly research hotspot in $\mathrm{T}$ lymphocytes, is still disputing. Some studies illustrate the downward number of both natural Tregs and induced Tregs in the aging process, while others found that the population of $\mathrm{CD} 4^{+}$Treg increased remarkably in immunosenescence $(12,13)$. Genetic and epigenetic modification also weakens the capacity of $\mathrm{T}$ cells (37). In all, complex cellular biological changes make the host susceptible to infection (37) and new technologies like single cell sequencing would discover a virgin land in this area and help researchers focus on the underlying mechanisms.

Senescent $\mathrm{T}$ cells could participate in inflammaging process as a double agent role, which is based on its immunosuppression function in the early stage and active function in late stage (38). First, loss of TCR function affects the contact of myeloid-derived suppressor cell (MDSC) responsible for inflammatory suppression $(38,39)$. In late stage, inflammatory suppression was impaired owing to the increase of $\mathrm{CD} 8^{+} \mathrm{CD} 28^{-}$Tregs because of their weaker inflammatory resolution and shorter lifespan compared with $\mathrm{CD} 8^{+} \mathrm{CD} 28^{+}$Tregs (14). Thymic atrophy impairs the negative selection, contributing to increasing self-antigenrecognizing conventional $\mathrm{T}$ cells (15). The imbalance of $\mathrm{T}$ cell subtypes causes the disorder of inflammatory factor production such as interferon-inducible protein-10, interleukin (IL)-6 and IL- 8 , increasing with age and exacerbate the inflammaging state $(16,17)$. The DNA damage resulting from telomere attrition in senescent $\mathrm{T}$ cell activates the NF- $\mathrm{BB}$ pathway, contributing to consistent uplifting of pro-inflammatory mediators (38).

Similarly with T cells, the aging changes of B cells include the reduction of naïve $B$ cells together with expansion of memory $B$ cells $(18,19)$. Senescent B cells with altered B cell receptors and functions are associated with higher cytokines production and antibody presentation (40). Besides, B cells under chronic inflammatory simulation tend to produce proinflammatory cytokine and pathogenic antibodies (20).
The innate immune system consists of several physical barriers and various cells, including monocytes/macrophages, neutrophils, eosinophils, basophils, dendritic cells and innatelike lymphocytes like NK cells (41). Although less attention has been paid, transformation of innate immunity is earlier and stronger than adaptive immunity during the senescent process, indicating its crucial role in the natural aging and pathology.

Monocytes, macrophages, dendritic cells (DCs) and microglia are responsible to chemotaxis, phagocytosis, secretion and antigen presentation, but these functions decline in elderly individuals. Those over 55 turned out to have more peripheral monocytes with impaired chemotaxis and phagocytosis. Moreover, the inflammatory pathway and immune response of macrophages are downregulated and suppressed $(21,22)$. The number of myeloid DCs and monocyte precursors progressively decreases with increasing age, though the number of classical $\mathrm{CD} 14^{+} \mathrm{CD} 16$ monocytes remains stable (27). A class of $\mathrm{CD} 14^{+} \mathrm{CD} 16^{+}$ monocyte subset, with downregulated expression of HLA-DR and CX3CR1, increases with age significantly $(23,24)$. Interestingly, some shifts result in the weaker defense ability, while other changes result in the permanent low-level inflammatory environment. The alteration of subtypes and upregulation of pro-inflammatory cytokine expression is present in both aging DCs and microglia, aggravating the inflammation in CNS (28-31). Besides, dysfunction of microRNA results in higher production of inflammatory cytokines such as IL-6, and reactive oxygen species (ROS), noted in both steady and active state of monocytes during aging (24-26).

Neutrophils, as an essential part of innate immunity performing cytotoxic effects and phagocytosis, are closely related to several inflammatory diseases. The function and lifespan of neutrophils are regulated by various cytokines: IL- 2 prolongs the lifespan and promotes inflammation while tumor necrosis factor (TNF)- $\alpha$ induces the apoptosis. Senescent neutrophils show decreased phagocytic, chemotactic ability and ROS production while the adherent ability appears to remain still $(38,42)$. Although these changes result in down-regulation of the inflammatory response, the increased neutrophil generation enhances the inflammaging (43). Overexpression of PI3K results in the inaccurate migration of neutrophils and damages of normal tissues (43, 44). Besides, elevated level of IL- 6 and IL-8 enhances neutrophils activation which would conversely affect inflammation outcome (45).

NK cells are differentiated from myeloid-lymphoid stem cells, and they perform unspecific killing function targeting abnormal cells like tumor cells, injured cells, and virus-infected cells via multiple cytotoxic effects. Commonly, senescent NK cells have weaker proliferative ability and larger cytotoxic subpopulation (33). When immune response initials, cytotoxicity of NK cells from elderly donors are weaker, which results from the alteration of receptors or production of enzymes (46). Further, NK cells were overactive in aging process via alteration of TLR function and shift of subtypes, and they act as effector in inflammaging (34).

\section{Immunosenescence Plays an Important Role in Age-related CNS Disease}

Immunosenescence is the core of various aged-related CNS diseases, including cerebral vessel diseases and degenerative 
neurological diseases. At the beginning of immunosenescence, senescent cells fail to be cleared, resulting in accumulation of abnormal cells and cell fragment and induction of SASP (47). On this basis, the systemic pro-inflammatory state damages brain tissue, which is worse owing to the pro-inflammatory mediators produced from SASP (Figure 1).

Alzheimer's disease (AD), the pathogenesis of which remains unknown, has a great connection with age. Most scientists believe in beta amyloid hypothesis that defines the deposition of $\beta$ amyloid peptide (A $\beta$ ) as initiator, interrupting the cellular metabolism and eventually progressing into $\mathrm{AD}$. Immunosenescence is also considered as a candidate mechanism of $\mathrm{AD}$, because the accumulation of $\mathrm{A} \beta$ is closely associated with impaired clearance ability and cytotoxic effect of senescent microglia (48). A $\beta$ deposition interacts with glial cells, pericytes, and neurons, modifies $\mathrm{BBB}$ and leads to immune cell infiltration (49). Those infiltrated cells and inflammatory mediators induce a proinflammatory environment around the lesion, which eventually leads to neuroinflammation and neurodegeneration (50,51). Current study concluded that the systematic immune response also involves in the neuroinflammation for which there is a large amount of evidence suggesting a proinflammatory state in $\mathrm{AD}$ patient, which implicates the complicated influence of immunosenescence in this disease (50).

Parkinson's disease (PD) is another common neurodegenerative disease in elderly that results from the degeneration of dopaminergic (DA) neurons in substantia nigra, leading to severe motor disorders. Mounting evidence suggested that oxidative stress and inflammation injure the DA neurons, in which microglia and astrocytes also play important roles (5254). Early study suggests that humoral immunity targets DA neurons which promotes the neuroinflammation and neurodegeneration in PD patient, agreeing with the activated microglia and elevated cytokines observed in neurotoxin-based PD model $(55,56)$. The therapy that focuses on balance between pro- and anti-inflammation provides a promising therapeutic strategy of PD (57). Pharmacological inhibition of oxidation and inflammation reverses the function of monoaminergic synthesis, which also supports this idea (58).

Cerebral vascular disease is a class of disease involving brain vessels, including vasculitis, cerebral amyloid angiopathy, subacute arteriosclerotic encephalopathy and CSVD, some of which have been prone to be associated with immunosenescence and inflammaging $(59,60)$. There lies a gap in CSVD, which we will discuss in the next section.

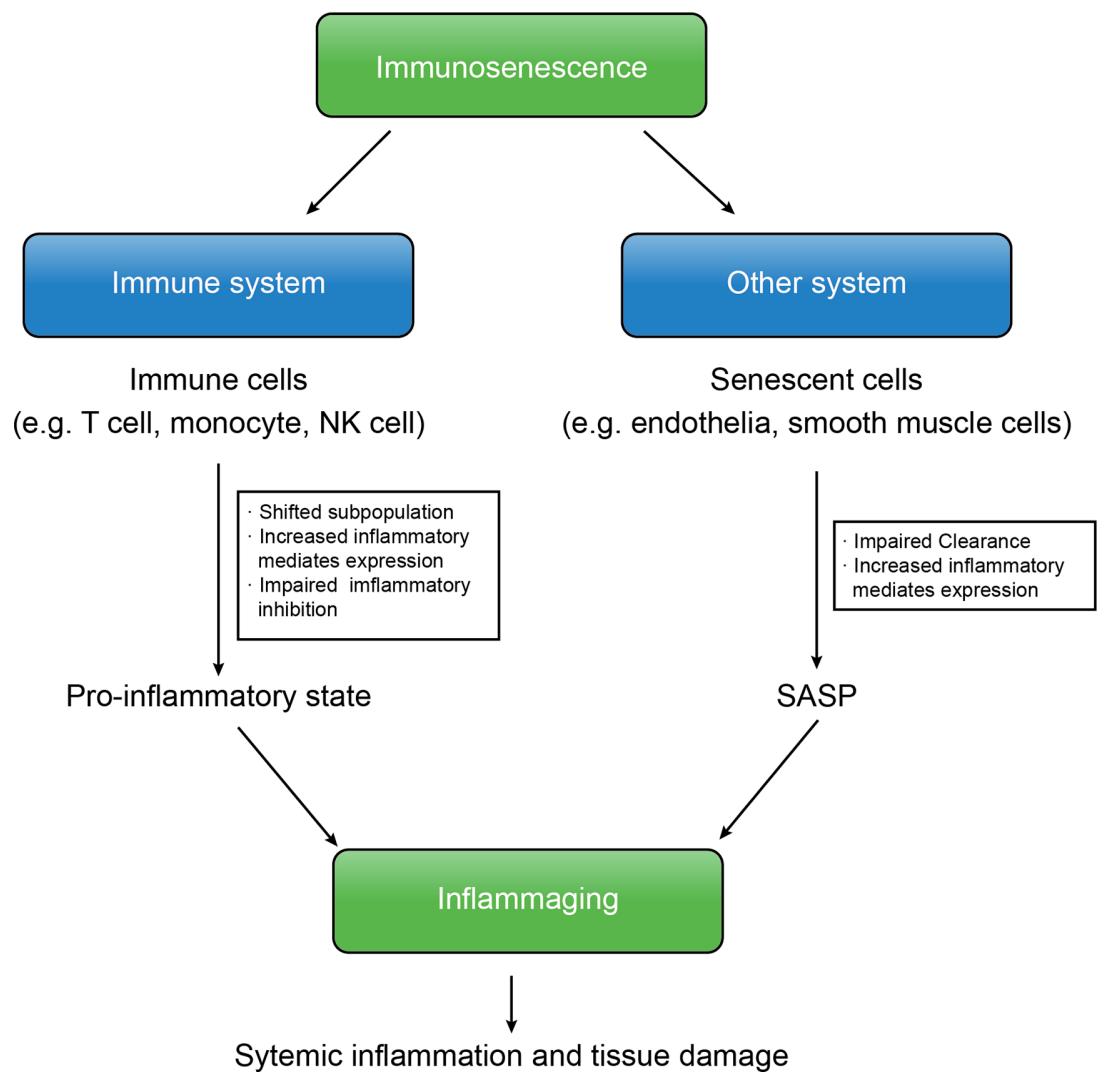

FIGURE 1 | The immunosenescence and inflammaging are interconnected. In immunosenescence, accumulation of senescent cells produce SASP, increased expression of inflammatory mediates of both immune cells and senescent cells and the insufficient downregulation of inflammation shift the body into the inflammaging state that results in further systemic inflammation and tissue damage, leading to several aged-related diseases. 


\section{SENESCENCE OF IMMUNE CELLS, NON- IMMUNE CELLS AND BBB DYSFUNCTION IN AGE-RELATED CSVD}

With ever-increasing life expectancy worldwide, the number of individuals living in the community with age-related diseases, especially CSVD (ArCSVD), will increase (61). The main manifestations of ArCSVD include stroke, cognitive declines, gait disorder, psychiatric disorders and urinary incontinence, and its sequelae would impose a considerable burden on families and society. ArCSVD is significantly associated with risk factors like aging, arterial hypertension, smoking, diabetes mellitus, obstructive sleep apnea (62-64). Besides, ArCSVD mainly affects the small blood vessel of the cortex or medulla, including small arteries, arterioles, capillaries, venules and small veins. The initial pathological characteristics are endothelial proliferation, small vessel wall thicken and arterial disorganization, and then develop into arteriolosclerosis and lipohyalinosis, which underline the pathological basis of ArCSVD. From an imaging perspective, ArCSVD is characterized by features like lacunar infarcts, white matter hyperintensity, subcortical infarcts, cerebral microbleedings, perivascular spaces, intracerebral hemorrhage and cerebral atrophy $(2,65,66)$. Unfortunately, pathological mechanisms of ArCSVD are incompletely understood yet. According to recent studies, immunosenescence roles in endothelial dysfunction and bloodbrain barrier disorder earn sustainable attention, which seems to be the possible candidate for further study $(67,68)$ (Figure 2).

\section{Senescence of Immune Cells}

Immune cells senescence in the early stage of ArCSVD intrinsically suppresses normal immune response and as a result increases infection risk, whereas the subsequent SASP would produce proinflammatory cytokines, including IL-1 1 , IL-6, and IL-8, and consequently accelerate and aggravate endothelial injury and BBB leakage. Each subsets of immune cells display specific function of immunosenescence in ArCSVD.

As one important type of phagocytes, microglia take responsibility for removing injured or dead neurons, glial cells and debris of the myelin sheath in CNS. Meanwhile such cells also act as a stabilizer of CNS structure and maintain BBB integrity and promote injured tissue recovery (69). However, accumulating evidence confirmed that microglia would become senescent and dysfunction, most likely contributing to low-grade inflammation in CNS and aged-related neurodegenerative diseases $(70,71)$. From the perspective mechanisms, hypofunction of TREM2-DAP12 and CX3CL1CX3CR1 axes plays a crucial role in the loss of phagocytosis and inflammatory modulation $(30,31)$. Senescent microglia suffers from the loss of responsiveness, migration and phagocytosis, leaving the accumulation of senescent cells and debris as a source of chronic inflammation that damage cerebrovascular structures and neurons $(71,72)$. Up-regulation of age-dependent inflammatory pathways are related to pro-inflammatory shift and increasing cytokine production of microglia $(73,74)$. The cross-talk among astrocytes, neutrophils, monocytes and macrophages, exacerbating the immune cell infiltration and inflammatory response consequently (75). In conclusion, senescent microglia activate inflammaging by tilting toward pro-inflammatory state, which is responsible for neuron degeneration and BBB leakage (76).

In case of infiltrated immune cells augmented in the early onset ArCSVD, including macrophages, neutrophils, T cells and NK cells, are responsible for the inflammation, endothelial dysfunction and ischemia of the area (77). As we mention above, the infiltration of senescent immune cells with increasing inflammatory mediates expression and altered function would aggravate the inflammation storm in regions of the lesion. ROS and other cytotoxic products from infiltrating immune cells enhance the oxidative stress of endothelial cells, resulting in further vessel tone dysfunction and vascular remodeling (78). While localized inflammation is a crucial damage to the brain, it is also essential that the peripheral immune response could aggravate the inflammation. According to a community-based study, systemic inflammation is related to white matter microstructural integrity among older adults (79). Furthermore, anti-endothelial antibodies were found in ArCSVD patients, implying the relationship between $\mathrm{B}$ cell activation and endothelial dysfunction (80).

Taken together, the senescence of immune cells have a strong connection with dysfunction of glia cells and endothelial cells. Non-immune cells under the damage of inflammatory environment finally become dysfunctional and promote the progress and development of ArCSVD.

\section{Senescence of Non-Immune Cells}

Senescent residential non-immune cells in CNS, including endothelial cells, astrocyte, pericytes, and oligodendrocytes, are also found to play crucial roles in destruction and dysfunction of BBB in ArCSVD. Moreover, these senescent cells can be recognized by immune cells and activate a further response.

Endothelium is an important system regulating vessel remodeling, vascular tone, balance of inflammatory and coagulation. Of note, it is a fact that endothelial dysfunction is attributed to be a key mechanism in ArCSVD (81). Apart from the increasing age, there is cogent evidence supporting that two interconnected mechanisms-cellular oxidative stress and low-grade inflammation - also contribute to endothelial senescence (82).

Endothelial dysfunction mainly reflects in vessel tone changes. Multiple factors are known as the contributor in vessel tone regulation, but eNOS-derived $\mathrm{NO}$ is usually considered to be the most important mechanism. NO plays an important role in relaxation of vascular smooth muscle cells and preservation of cerebral blood flow (82). The inhibitory effect of NO restrains platelets from aggregation and adhesion, and the release of plateletderived growth factor that stimulates smooth muscle cell proliferation (83). Meanwhile, $\mathrm{NO}$ also prevents relating immune cells from activation of NF- $\mathrm{KB}$ and formation of inflammatory factors $(84,85)$. However, the changes of eNOS signaling on transcriptional and post-transcriptional level resulting from hypertension, angiotensin II and aging, suppress the function of eNOS by reducing NO synthesis (86-88). The loss of NO synthesis results in vessel tone disorder, reduces the cerebral blood flow, exacerbates oxidative stress and vulnerability of acute ischemia. Furthermore, there is a vicious cycle in the senescent endothelium (89). Activating TLR-NF- $\kappa B$ pathway results in subsequently 


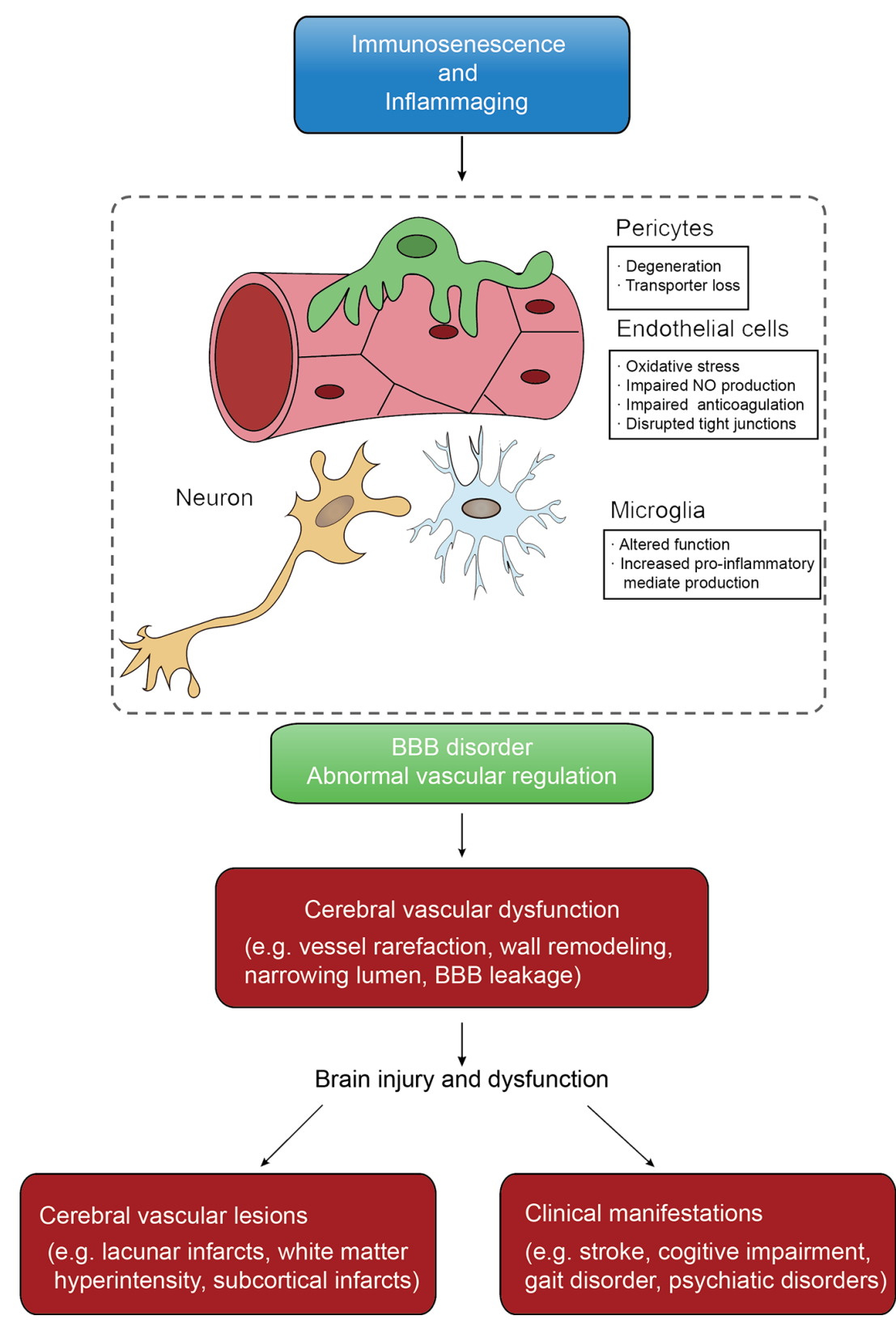

FIGURE 2 | The conceptual model of immunosenescence and inflammaging mechanism of CSVD. Immunosenescence and inflammaging affect both vascular function and BBB intactness that are keys of CSVD initiation. Cerebral vascular dysfunctions are various that further lead to pathological changes that can be found on neuroimaging and clinical features.

cytokines production including IL- $1 \alpha$, IL-1 $\beta$, IL-6, IL-8, IL-10, IL-12, TNF- $\alpha$, and IFN- $\gamma(25,90,91)$. Pro-inflammatory cytokines as well as the NF- $\mathrm{KB}$ protein induces the expression of NADPH oxidase, contributing to the increase production of ROS (92). These oxidative stress productions act as a positive feedback that increases NF- $\mathrm{KB}$ activity, meanwhile they also activate circulating and residential immune cells (89). Free radicals would impair NO production by causing eNOS uncoupling directly, the amplifying oxidative stress further worsening the dysregulation of NO production (93). Taken together, it's trapping in a vicious cycle of oxidative stress and inflammation that progressively damage the endothelial function which consequently develops into cerebrovascular dysfunction.

Pathologically, senescent epithelium results in impairment of proliferation and angiogenesis, leading to abnormal vessel remodelling (94). Vessel rarefaction is commonly observed in hypertension and ARCSVD patient, suggesting the reduction of the blood vessel in tissues (95). Decreased density and length of capillary are found in the cerebral cortex of aging people. Moreover, morpho-functional changes of capillary bed of the 
cortex, reduction of external diameter, and increased wall-tolumen ratio and resistance are found in hypertensive patients $(96,97)$. The consequences of cerebrovascular microstructure remodeling are ischemic changes in the regions supplied by the responsible blood vessels, showing as lacunar infarcts, white matter hyperintensity or perivascular space in imageology.

Pericytes are isolated contractile cells that regulate cerebral blood flow and maintain BBB (98). Pericytes participate in the formation of capillary basement membrane, while multiple signaling pathways between pericytes and astrocytes also exert an essential effect on BBB integrity $(99,100)$. Dysfunction of pericytes contributes to the aged-related cerebrovascular diseases. According to a current study, the loss of pericyte coverage is reported in aged mice, which may result from hypertensive induced oxidative stress (101). While another study found that the loss of pericyte in aging mice and attributed it to the glutamine pathway and ischemia as pericyte is sensitive to ischemia (102). Moreover, pericytes are also under the effect of inflammation. For example, some investigators observed increasing permeability of BBB resulting from IL-1 $\beta$ induced pericytes, and come to the conclusion that dysfunction of pericytes and inflammation may damage the integrity of BBB (103). What's more, loss of pericyte coverage reduces microcirculation, breaks down $\mathrm{BBB}$, exacerbates oxidative stress and cause neurodegeneration, which is observed in an experiment of pericyte-deficient mice (99). Thus, pericyte dysfunction is a crucial part should not be neglected in ArCSVD.

\section{Dysfunction of BBB}

It is obvious that $\mathrm{BBB}$ disorder, on the basis of endothelial dysfunction and pericyte disorder, plays a critical role in ArCSVD. BBB is composed of basic membrane, astrocytes end feet, pericytes and endothelial cells with tight junction (68). The incidence of $\mathrm{BBB}$ leakage increases with risk factors like toxicity, trauma, age and hypertension, and such injury is mainly related to cell response induced by immunosenescence $(104,105)$. Although the pathophysiology of BBB is complicated and intricate, the major process nevertheless is recognized, and the most identical initiation is endothelial dysfunction whose commonest outcome is BBB.

In the context of cerebrovascular dysregulation, chronic exposition of high shear force leads to the alteration of the tight junction and increased permeability of BBB (106). As a result, immune cells and plasma components could enter the brain parenchyma and trigger sterile inflammation (107). Subsequently, oxidative stress and inflammatory cytokines induced by the immune cells aggravate the local inflammatory response and hinder the damage repair $(85,108)$. Furthermore, damage of other glial cells and its production of large quantities of inflammatory mediators and toxic substances could destroy the barrier system (109).

Accordingly, a broad range of pathophysiological changes in $\mathrm{BBB}$ disorder would finally contribute to white matter lesions and other secondary damage in CSVD while imaging shows a phenomenon termed as white matter hyperintensities (WMH) in the clinical context (110). The degree of BBB leakage is commonly assessed according to the signal of $\mathrm{WMH}$ - the greater the range of $\mathrm{WMH}$, the more severe is the BBB leakage (111).

\section{CANDIDATE MARKERS OF IMMUNOSENESCENCE IN ARCSVD FOR CLINICAL DIAGNOSIS}

The clinical performance of ArCSVD is complicated and variable, and diagnosis of such disease relies on the imaging findings. However, imaging exists an inherent drawback that only the late stage patient whose injury is mostly irrecoverable could perform remarkable imaging manifestations, indicating an unmet need for developing one or more neo-markers for screening patients at early stage. Thereafter, immunosenescence is consist of a wide range of biomarkers alteration in the circulation system and shows its great potential in prediction of ArCSVD as a sensitive and accurate index available.

When ArCSVD initials and develops, multiple substances in circulation system experience drastic change. Inflammationrelative molecules, such as coding proteins and metabolites, have been the hotspot in this area. Several endothelial markers, involved in the activation of endothelial dysfunction and inflammation, were associated with the seriousness of CSVD, including neopterin, sICAM-1, and sVCAM-1 (112). Cytokines are another big family having great changes during the development of ArCSVD and divided into pro-inflammatory and anti-inflammatory subtypes based on their distinct functions. IL- 6 and TNF $\alpha$ have raised great attention as their pro-inflammatory function in ArCSVD. The uplifting of serum IL-6 was usually observed in aged-related disease, and it was prone to be associated with the progression of ArCSVD (113). Other inflammatory factors, like TNF- $\alpha$, were also increased in ArCSVD. It should be noted that the downward of antiinflammatory cytokines also makes sense in ArCSVD as the newly discoveries in IL-10 $(77,114)$.

Obviously, patterns and related metabolites were also seriously affected by ArCSVD. The most significant changes are involving glycol metabolism, lipid metabolism, vitamin B and vitamin D metabolism pathways as what is tested in clinical practice. Glycosylated hemoglobin (HbA1c), low density lipoprotein (LDL), homocysteine (Hcy), 25-hydroxyvitamin D3 [25(OH)D3] stand for the representatives for the foregoing pathways and the first three substances significantly upregulates in ArCSVD while the last one as the sole protective factor decreases.

Immune cells also act a crucial role in ArCSVD and the detection of these cells helps physicians and specialists better predict this disease. The progressive remodeling of immune system displays as the shifts of cell subtypes, including NK cells, $\mathrm{T}$ cells and $\mathrm{B}$ cells subpopulations $(115,116)$. The diminishing of naive $\mathrm{T}$ cell pool and increasing memory $\mathrm{T}$ cells may be the predictor of immunosenescence.

Today increasing evidence supports that non-coding RNAs have a great impact on development of ArCSVD. As the most well-known non-coding RNA, microRNAs have a more definitive function in a related area. MicroRNAs are a group of molecules that regulate the gene expression, mostly in a negative direction, and build a complicated interactive network in both pro- and anti-inflammatory pathways. Inflammation-associated microRNAs represent the conditions of immunosenescence such 
as MiR-126, MiR-146a, belonging to inflammatory pathway while also related to endothelial dysfunction, can be used for detection of ArCSVD $(117,118)$.

\section{THE POTENTIAL REJUVENATION STRATEGIES TO PREVENT CSVD DURING AGING}

Immunosenescence accelerates and exacerbates CSVD while CSVD conversely promotes senescence of either CNS resident cells or immune cells. Thereafter, it is meaningful to prevent CSVD by postponing the process of immunosenescence. As inflammation is the crucial part of immunosenescence that damage the structure and function of nervous system in ArCSVD patients, most studies focus on anti-inflammation treatment.

Targeting key immune cells can be a potential approach to prevent or improve ArCSVD. Selectively inhibiting the activation or cytokines secretion of microglia and macrophages is a potential method to prevent the overactive neuroinflammation (119). Some studies found that regulating the gene expression and altering the phenotypes of microglia diminished the inflammation and promoted the recovery by drugs or chemotactic factors (120, 121). Besides, regaining the loss functions of immune cells can be another approach to prevent excessive inflammation. To clear the senescent cells, enhancement of phagocytosis in macrophages and chemotaxis in other immune cells and impeding the overproduction of pro-inflammatory cytokines are out of urgent necessity (122). In addition, the differentiation of Tregs, as well as its enhanced anti-inflammatory features, could be a prospective way to delay the progress of ArCSVD. Overall, preventing ArCSVD is still at the preclinical stage and not currently accepted in patients. Prior to clinical manifestations, further studies are required for better understanding the mechanism of interaction between antiinflammation and ArCSVD treatment.

Actually, clinical practitioners reach a common consensus that lifestyle interventions may be a considerable and effective approach to alleviating CNS damage. The lifestyle intervention is well tolerated and it contains the management of sleep, motion and diet. The risk factors like hypertension, hyperlipidemia, hyperglycemia should be also controlled as they can disturb both the metabolism and inflammaging $(82,123,124)$. Nutrition supplement, such as zinc, docosahexaenoic acid, active vitamin D,

\section{REFERENCES}

1. Mishra A, Chauhan G, Violleau MH, Vojinovic D, Jian X, Bis JC, et al. Association of variants in HTRA1 and NOTCH3 with MRI-defined extremes of cerebral small vessel disease in older subjects. Brain (2019) 142(4):1009-23. doi: 10.1093/brain/awz024

2. Pantoni L. Cerebral small vessel disease: from pathogenesis and clinical characteristics to therapeutic challenges. Lancet Neurol (2010) 9(7):689-701. doi: 10.1016/S1474-4422(10)70104-6

3. Rensma SP, van Sloten TT, Launer LJ, Stehouwer CDA. Cerebral small vessel disease and risk of incident stroke, dementia and depression, and allcause mortality: A systematic review and meta-analysis. Neurosci Biobehav $\operatorname{Rev}$ (2018) 90:164-73. doi: 10.1016/j.neubiorev.2018.04.003 mecobalamin and folic acid, is also beneficial to the control of inflammation and immune response (125).

\section{CONCLUSION}

CSVD is closely related to aging and little interest was shown in the contribution of immunity to CSVD according to previous studies. Nonetheless, the immunosenescence could be a prominent participator in the initiation and development of ArCSVD. So far, more studies should be carried out to further understand the association of immunosenescence and CSVD:(a) Whether the senescence of endothelia earlier than immunosenescence or not? (b) How immunosenescence disrupts blood-brain barrier step by step in this disease? (c) What should we do to uncover the specific targets connecting immunosenescence with CSVD under the rapid development of bioinformatic analysis and related technologies for sequencing? In all, the researchers take the responsibility to solve these questions and better transform the fundamental studies to the clinical practice.

\section{AUTHOR CONTRIBUTIONS}

BJ wrote the manuscript. $\mathrm{MH}$ and WC conducted the manuscript editing. BZ and ZL designed and critically revised the manuscript, supervised the project, and obtained fundings for this study. All authors contributed to the article and approved the submitted version.

\section{FUNDING}

This work was supported by Guangzhou Science and Technology Program key project (202007030010), National Natural Science Foundation of China (81971110 to ZL) and the Guangdong Basic and Applied Basic Research Foundation (2020A1515010056).

\section{ACKNOWLEDGMENTS}

We want to sincerely extend our thanks to Dr. Xining Wang (Sun Yat-sen Memorial Hospital) for his constant tolerance and hard work for language editing.

4. Weyand CM, Goronzy JJ. Aging of the Immune System. Mechanisms and Therapeutic Targets. Ann Am Thoracic Soc (2016) 13:S422-8. doi: 10.1513/ AnnalsATS.201602-095AW

5. Fu Y, Yan Y. Emerging Role of Immunity in Cerebral Small Vessel Disease. Front Immunol (2018) 9:67. doi: 10.3389/fimmu.2018.00067

6. Linton P, Thoman ML. Immunosenescence in monocytes, macrophages, and dendritic cells: Lessons learned from the lung and heart. Immunol Lett (2014) 162(1):290-7. doi: 10.1016/j.imlet.2014.06.017

7. Erickson M, Banks W. Age-Associated Changes in the Immune System and BloodBrain Barrier Functions. Int J Mol Sci (2019) 20(7):1632. doi: 10.3390/ijms20071632

8. Pangrazzi L, Meryk A, Naismith E, Koziel R, Lair J, Krismer M, et al. "Inflamm-aging" influences immune cell survival factors in human bone marrow. Eur J Immunol (2017) 47(3):481-92. doi: 10.1002/eji.201646570 
9. Alam R. A brief review of the immune system. Prim Care (1998) 25(4):72738. doi: 10.1016/s0095-4543(05)70084-1

10. Kilpatrick RD, Rickabaugh T, Hultin LE, Hultin P, Hausner MA, Detels R, et al. Homeostasis of the Naive CD4+ T Cell Compartment during Aging. J Immunol (2008) 180(3):1499-507. doi: 10.4049/jimmunol.180.3.1499

11. Li M, Yao D, Zeng X, Kasakovski D, Zhang Y, Chen S, et al. Age related human T cell subset evolution and senescence. Immun Ageing (2019) 16 (1):24. doi: 10.1186/s12979-019-0165-8

12. Jagger A, Shimojima Y, Goronzy JJ, Weyand CM. Regulatory T cells and the immune aging process: a mini-review. Gerontology (2014) 60(2):130-7. doi: $10.1159 / 000355303$

13. Raynor J, Lages CS, Shehata H, Hildeman DA, Chougnet CA. Homeostasis and function of regulatory T cells in aging. Curr Opin Immunol (2012) 24 (4):482-7. doi: 10.1016/j.coi.2012.04.005

14. Fessler J, Raicht A, Husic R, Ficjan A, Schwarz C, Duftner C, et al. Novel Senescent Regulatory T-Cell Subset with Impaired Suppressive Function in Rheumatoid Arthritis. Front Immunol (2017) 8:300. doi: 10.3389/ fimmu.2017.00300

15. Thomas R, Wang W, Su DM. Contributions of Age-Related Thymic Involution to Immunosenescence and Inflammaging. Immun Ageing (2020) 17:2. doi: 10.1186/s12979-020-0173-8

16. Hu WT, Howell JC, Ozturk T, Gangishetti U, Kollhoff AL, Hatcher-Martin JM, et al. CSF Cytokines in Aging, Multiple Sclerosis, and Dementia. Front Immunol (2019) 10:480. doi: 10.3389/fimmu.2019.00480

17. Singh $\mathrm{T}$, Newman $\mathrm{AB}$. Inflammatory markers in population studies of aging. Ageing Res Rev (2011) 10(3):319-29. doi: 10.1016/j.arr.2010.11.002

18. Bulati M, Caruso C, Colonna-Romano G. From lymphopoiesis to plasma cells differentiation, the age-related modifications of B cell compartment are influenced by "inflamm-ageing". Ageing Res Rev (2017) 36:125-36. doi: 10.1016/j.arr.2017.04.001

19. Palma P, Rinaldi S, Cotugno N, Santilli V, Pahwa S, Rossi P, et al. Premature B-cell senescence as a consequence of chronic immune activation. Hum Vaccin Immunother (2014) 10(7):2083-8. doi: 10.4161/hv.28698

20. Frasca D, Blomberg BB. Adipose Tissue Inflammation Induces B Cell Inflammation and Decreases B Cell Function in Aging. Front Immunol (2017) 8:1003. doi: 10.3389/fimmu.2017.01003

21. Antonaci S, Jirillo E, Ventura MT, Garofalo AR, Bonomo L. Non-specific immunity in aging: deficiency of monocyte and polymorphonuclear cellmediated functions. Mech Ageing Dev (1984) 24(3):367-75. doi: 10.1016/ 0047-6374(84)90121-0

22. Mahbub S, Deburghgraeve CR, Kovacs EJ. Advanced age impairs macrophage polarization. J Interferon Cytokine Res (2012) 32(1):18-26. doi: $10.1089 /$ jir. 2011.0058

23. Seidler S, Zimmermann HW, Bartneck M, Trautwein C, Tacke F. Agedependent alterations of monocyte subsets and monocyte-related chemokine pathways in healthy adults. BMC Immunol (2010) 11:30. doi: 10.1186/1471-2172-11-30

24. Sadeghi HM, Schnelle JF, Thoma JK, Nishanian P, Fahey JL. Phenotypic and functional characteristics of circulating monocytes of elderly persons. Exp Gerontol (1999) 34(8):959-70. doi: 10.1016/s0531-5565(99)00065-0

25. Olivieri F, Rippo MR, Prattichizzo F, Babini L, Graciotti L, Recchioni R, et al. Toll like receptor signaling in "inflammaging": microRNA as new players. Immun Ageing (2013) 10(1):11. doi: 10.1186/1742-4933-10-11

26. Jacinto TA, Meireles GS, Dias AT, Aires R, Porto ML, Gava AL, et al. Increased ROS production and DNA damage in monocytes are biomarkers of aging and atherosclerosis. Biol Res (2018) 51(1):33. doi: 10.1186/s40659018-0182-7

27. Della BS, Bierti L, Presicce P, Arienti R, Valenti M, Saresella M, et al. Peripheral blood dendritic cells and monocytes are differently regulated in the elderly. Clin Immunol (2007) 122(2):220-8. doi: 10.1016/ j.clim.2006.09.012

28. Splunter MV, Perdijk O, Fick-Brinkhof H, Floris-Vollenbroek EG, Meijer B, Brugman S, et al. Plasmacytoid dendritic cell and myeloid dendritic cell function in ageing: A comparison between elderly and young adult women. PloS One (2019) 14(12):e225825. doi: 10.1371/journal.pone.0225825

29. Yao K, Zhao YF. Aging modulates microglia phenotypes in neuroinflammation of MPTP-PD mice. Exp Gerontol (2018) 111:86-93. doi: 10.1016/j.exger. 2018.07.010
30. Mecca C, Giambanco I, Donato R, Arcuri C. Microglia and Aging: The Role of the TREM2-DAP12 and CX3CL1-CX3CR1 Axes. Int J Mol Sci (2018) 19 (1):318. doi: 10.3390/ijms19010318

31. Vida C, Gonzalez EM, De la Fuente M. Increase of oxidation and inflammation in nervous and immune systems with aging and anxiety. Curr Pharm Des (2014) 20(29):4656-78. doi: 10.2174/138161282066614 0130201734

32. Niwa Y, Kasama T, Miyachi Y, Kanoh T. Neutrophil chemotaxis, phagocytosis and parameters of reactive oxygen species in human aging: cross-sectional and longitudinal studies. Life Sci (1989) 44(22):1655-64. doi: 10.1016/0024-3205(89)90482-7

33. Gounder SS, Abdullah B, Radzuanb N, Zain F, Sait N, Chua C, et al. Effect of Aging on NK Cell Population and Their Proliferation at Ex Vivo Culture Condition. Anal Cell Pathol (Amst) (2018) 2018:7871814. doi: 10.1155/2018/ 7871814

34. Camous X, Pera A, Solana R, Larbi A. NK cells in healthy aging and ageassociated diseases. J BioMed Biotechnol (2012) 2012:195956. doi: 10.1155/ 2012/195956

35. Goronzy JJ, Fang F, Cavanagh MM, Qi Q, Weyand CM. Naive T Cell Maintenance and Function in Human Aging. J Immunol (2015) 194 (9):4073-80. doi: 10.4049/jimmunol.1500046

36. Le Page A, Dupuis G, Larbi A, Witkowski JM, Fülöp T. Signal transduction changes in $\mathrm{CD} 4+$ and $\mathrm{CD} 8+\mathrm{T}$ cell subpopulations with aging. Exp Gerontol (2018) 105:128-39. doi: 10.1016/j.exger.2018.01.005

37. Tu W, Rao S. Mechanisms Underlying T Cell Immunosenescence: Aging and Cytomegalovirus Infection. Front Microbiol (2016) 7:2111. doi: 10.3389/ fmicb.2016.02111

38. Olivieri F, Albertini MC, Orciani M, Ceka A, Cricca M, Procopio AD, et al. DNA damage response (DDR) and senescence: shuttled inflamma-miRNAs on the stage of inflamm-aging. Oncotarget (2015) 6(34):35509-21. doi: 10.18632/oncotarget.5899

39. Alves AS, Bueno V. Immunosenescence: participation of T lymphocytes and myeloid-derived suppressor cells in aging-related immune response changes. Einstein (Sao Paulo) (2019) 17(2):B4733. doi: 10.31744/einstein_journal/ 2019RB4733

40. Ma S, Wang C, Mao X. Hao Y. B Cell Dysfunction Associated With Aging and Autoimmune Diseases. Front Immunol (2019) 10:318. doi: 10.3389/ fimmu.2019.00318

41. Riera RM, Perez-Martinez D, Castillo FC. Innate immunity in vertebrates: an overview. Immunology (2016) 148(2):125-39. doi: 10.1111/imm.12597

42. Wenisch C, Patruta S, Daxbock F, Krause R, Horl W. Effect of age on human neutrophil function. J Leukoc Biol (2000) 67(1):40-5. doi: 10.1002/jlb.67.1.40

43. Salminen A, Kaarniranta K, Kauppinen A. The role of myeloid-derived suppressor cells (MDSC) in the inflammaging process. Ageing Res Rev (2018) 48:1-10. doi: 10.1016/j.arr.2018.09.001

44. Sapey E, Greenwood H, Walton G, Mann E, Love A, Aaronson N, et al. Phosphoinositide 3-kinase inhibition restores neutrophil accuracy in the elderly: toward targeted treatments for immunosenescence. Blood (2014) 123 (2):239-48. doi: 10.1182/blood-2013-08-519520

45. Roy-O'Reilly MA, Ahnstedt H, Spychala MS, Munshi Y, Aronowski J, Sansing LH, et al. Aging exacerbates neutrophil pathogenicity in ischemic stroke. Aging (Albany NY) (2020) 12(1):436-61. doi: 10.18632/aging.102632

46. Mocchegiani E, Malavolta M. NK and NKT cell functions in immunosenescence. Aging Cell (2004) 3(4):177-84. doi: 10.1111/j.14749728.2004.00107.x

47. Denkinger MD, Leins H, Schirmbeck R, Florian MC, Geiger H. HSC Aging and Senescent Immune Remodeling. Trends Immunol (2015) 36(12):815-24. doi: 10.1016/j.it.2015.10.008

48. Hansen DV, Hanson JE, Sheng M. Microglia in Alzheimer's disease. J Cell Biol (2018) 217(2):459-72. doi: 10.1083/jcb.201709069

49. Zenaro E, Piacentino G, Constantin G. The blood-brain barrier in Alzheimer's disease. Neurobiol Dis (2017) 107:41-56. doi: 10.1016/j.nbd.2016.07.007

50. Martorana A, Bulati M, Buffa S, Pellicano M, Caruso C, Candore G, et al. Immunosenescence, inflammation and Alzheimer's disease. Longev Healthspan (2012) 1:8. doi: 10.1186/2046-2395-1-8

51. Trushina E. Alzheimer's disease mechanisms in peripheral cells: Promises and challenges. Alzheimers Dement (N Y) (2019) 5:652-60. doi: 10.1016/ j.trci.2019.06.008 
52. Ariga H, Takahashi-Niki K, Kato I, Maita H, Niki T, Iguchi-Ariga SM. Neuroprotective function of DJ-1 in Parkinson's disease. Oxid Med Cell Longev (2013) 2013:683920. doi: 10.1155/2013/683920

53. Saito Y. Oxidized DJ-1 as a possible biomarker of Parkinson's disease. J Clin Biochem Nutr (2014) 54(3):138-44. doi: 10.3164/jcbn.13-108

54. Rozycka A, Jagodzinski PP, Kozubski W, Lianeri M, Dorszewska J. Homocysteine Level and Mechanisms of Injury in Parkinson's Disease as Related to MTHFR, MTR, and MTHFD1 Genes Polymorphisms and LDopa Treatment. Curr Genomics (2013) 14(8):534-42. doi: 10.2174/ 1389202914666131210210559

55. Orr CF, Rowe DB, Mizuno Y, Mori H, Halliday GM. A possible role for humoral immunity in the pathogenesis of Parkinson's disease. Brain (2005) 128(Pt 11):2665-74. doi: 10.1093/brain/awh625

56. Gelders G, Baekelandt V, Van der Perren A. Linking Neuroinflammation and Neurodegeneration in Parkinson's Disease. J. Immunol Res (2018) 2018:4784268. doi: 10.1155/2018/4784268

57. Calabrese V, Santoro A, Monti D, Crupi R, Di Paola R, Latteri S, et al. Aging and Parkinson's Disease: Inflammaging, neuroinflammation and biological remodeling as key factors in pathogenesis. Free Radic Biol Med (2018) 115:80-91. doi: 10.1016/j.freeradbiomed.2017.10.379

58. Sarubbo F, Ramis MR, Kienzer C, Aparicio S, Esteban S, Miralles A, et al. Chronic Silymarin, Quercetin and Naringenin Treatments Increase Monoamines Synthesis and Hippocampal Sirtl Levels Improving Cognition in Aged Rats. J Neuroimmune Pharmacol (2018) 13(1):24-38. doi: 10.1007/s11481-017-9759-0

59. Pletz MW, Duda PW, Kappos L, Steck AJ. Immune-mediated neuropathies: etiology and pathogenic relationship to aging processes. J Neuroimmunol (2003) 137(1-2):1-11. doi: 10.1016/s0165-5728(03)00010-9

60. Corovic A, Kelly S, Markus HS. Cerebral amyloid angiopathy associated with inflammation: A systematic review of clinical and imaging features and outcome. Int J Stroke (2018) 13(3):257-67. doi: 10.1177/1747493017741569

61. Hilal S, Mok V, Youn YC, Wong A, Ikram MK, Chen CL. Prevalence, risk factors and consequences of cerebral small vessel diseases: data from three Asian countries. J Neurol Neurosurg Psychiatry (2017) 88(8):669-74. doi: 10.1136/jnnp-2016-315324

62. Khan U, Porteous L, Hassan A, Markus HS. Risk factor profile of cerebral small vessel disease and its subtypes. J Neurol Neurosurg Psychiatry (2007) 78 (7):702-6. doi: 10.1136/jnnp.2006.103549

63. Huang Y, Yang C, Yuan R, Liu M, Hao Z. Association of obstructive sleep apnea and cerebral small vessel disease: a systematic review and metaanalysis. Sleep (2019) 43(4):zsz264. doi: 10.1093/sleep/zsz264

64. Han F, Zhai FF, Wang Q, Zhou LX, Ni J, Yao M, et al. Prevalence and Risk Factors of Cerebral Small Vessel Disease in a Chinese Population-Based Sample. J Stroke (2018) 20(2):239-46. doi: 10.5853/jos.2017.02110

65. Fisher CM. Lacunar strokes and infarcts: a review. Neurology (1982) 32 (8):871-6. doi: 10.1212/wnl.32.8.871

66. Wardlaw JM, Smith EE, Biessels GJ, Cordonnier C, Fazekas F, Frayne R, et al. Neuroimaging standards for research into small vessel disease and its contribution to ageing and neurodegeneration. Lancet Neurol (2013) 12 (8):822-38. doi: 10.1016/S1474-4422(13)70124-8

67. Regenhardt RW, Das AS, Lo EH, Caplan LR. Advances in Understanding the Pathophysiology of Lacunar Stroke: A Review. JAMA Neurol (2018) 75 (10):1273-81. doi: 10.1001/jamaneurol.2018.1073

68. Wardlaw JM, Smith C, Dichgans M. Mechanisms of sporadic cerebral small vessel disease: insights from neuroimaging. Lancet Neurol (2013) 12(5):48397. doi: 10.1016/S1474-4422(13)70060-7

69. Wolf SA, Boddeke HW, Kettenmann H. Microglia in Physiology and Disease. Annu Rev Physiol (2017) 79:619-43. doi: 10.1146/annurevphysiol-022516-034406

70. Niraula A, Sheridan JF, Godbout JP. Microglia Priming with Aging and Stress. Neuropsychopharmacol (2017) 42(1):318-33. doi: 10.1038/npp.2016.185

71. Plaza-Zabala A, Sierra-Torre V, Sierra A. Autophagy and Microglia: Novel Partners in Neurodegeneration and Aging. Int J Mol Sci (2017) 18(3):598. doi: 10.3390/ijms18030598

72. Harry GJ. Microglia during development and aging. Pharmacol Ther (2013) 139(3):313-26. doi: 10.1016/j.pharmthera.2013.04.013

73. Kirkland JL, Tchkonia T. Cellular Senescence: A Translational Perspective. Ebiomedicine (2017) 21:21-8. doi: 10.1016/j.ebiom.2017.04.013
74. Ventura MT, Casciaro M, Gangemi S, Buquicchio R. Immunosenescence in aging: between immune cells depletion and cytokines up-regulation. Clin Mol Allergy (2017) 15:21. doi: 10.1186/s12948-017-0077-0

75. Ransohoff RM, Brown MA. Innate immunity in the central nervous system. J Clin Invest (2012) 122(4):1164-71. doi: 10.1172/JCI58644

76. Ronaldson PT, Davis TP. Blood-brain barrier integrity and glial support: mechanisms that can be targeted for novel therapeutic approaches in stroke. Curr Pharm Des (2012) 18(25):3624-44. doi: 10.2174/138161212802002625

77. Kaiser D, Weise G, Moller K, Scheibe J, Posel C, Baasch S, et al. Spontaneous white matter damage, cognitive decline and neuroinflammation in middle-aged hypertensive rats: an animal model of early-stage cerebral small vessel disease. Acta Neuropathol Commun (2014) 2:169. doi: 10.1186/s40478-014-0169-8

78. De Silva TM, Miller AA. Cerebral Small Vessel Disease: Targeting Oxidative Stress as a Novel Therapeutic Strategy? Front Pharmacol (2016) 7:61. doi: 10.3389/fphar.2016.00061

79. Walker KA, Power MC, Hoogeveen RC, Folsom AR, Ballantyne CM, Knopman DS, et al. Midlife Systemic Inflammation, Late-Life White Matter Integrity, and Cerebral Small Vessel Disease: The Atherosclerosis Risk in Communities Study. Stroke (2017) 48(12):3196-202. doi: 10.1161/ STROKEAHA.117.018675

80. Kimura A, Sakurai T, Yamada M, Koumura A, Hayashi Y, Tanaka Y, et al. Anti-endothelial cell antibodies in patients with cerebral small vessel disease. Curr Neurovasc Res (2012) 9(4):296-301. doi: 10.2174/156720212803530726

81. Poggesi A, Pasi M, Pescini F, Pantoni L, Inzitari D. Circulating biologic markers of endothelial dysfunction in cerebral small vessel disease: A review. J Cereb Blood Flow Metab (2016) 36(1):72-94. doi: 10.1038/jcbfm.2015.116

82. Buford TW. Hypertension and aging. Ageing Res Rev (2016) 26:96-111. doi: 10.1016/j.arr.2016.01.007

83. Li H, Forstermann U. Nitric oxide in the pathogenesis of vascular disease. J Pathol (2000) 190(3):244-54. doi: 10.1002/(SICI)1096-9896(200002) 190:3<244::AID-PATH575<3.0.CO;2-8

84. Yurdagul AJ, Chen J, Funk SD, Albert P, Kevil CG, Orr AW. Altered nitric oxide production mediates matrix-specific PAK2 and NF-kappaB activation by flow. Mol Biol Cell (2013) 24(3):398-408. doi: 10.1091/mbc.E12-07-0513

85. Hu X, De Silva TM, Chen J, Faraci FM. Cerebral Vascular Disease and Neurovascular Injury in Ischemic Stroke. Circ Res (2017) 120(3):449-71. doi: 10.1161/CIRCRESAHA.116.308427

86. McCarty MF. NADPH Oxidase Activity in Cerebral Arterioles Is a Key Mediator of Cerebral Small Vessel Disease-Implications for Prevention. Healthcare (Basel) (2015) 3(2):233-51. doi: 10.3390/healthcare3020233

87. Berkowitz DE, White R, Li D, Minhas KM, Cernetich A, Kim S, et al. Arginase reciprocally regulates nitric oxide synthase activity and contributes to endothelial dysfunction in aging blood vessels. Circulation (2003) 108 (16):2000-6. doi: 10.1161/01.CIR.0000092948.04444.C7

88. Vanhoutte PM, Zhao Y, Xu A, Leung SW. Thirty Years of Saying NO: Sources, Fate, Actions, and Misfortunes of the Endothelium-Derived Vasodilator Mediator. Circ Res (2016) 119(2):375-96. doi: 10.1161/CIRCRESAHA.116.306531

89. Donato AJ, Morgan RG, Walker AE, Lesniewski LA. Cellular and molecular biology of aging endothelial cells. J Mol Cell Cardiol (2015) 89(Pt B):122-35. doi: 10.1016/j.yjmcc.2015.01.021

90. Mun GI, Boo YC. Identification of CD44 as a senescence-induced cell adhesion gene responsible for the enhanced monocyte recruitment to senescent endothelial cells. Am J Physiol Heart Circ Physiol (2010) 298(6): H2102-11. doi: 10.1152/ajpheart.00835.2009

91. Ungvari Z, Tarantini S, Donato AJ, Galvan V, Csiszar A. Mechanisms of Vascular Aging. Circ Res (2018) 123(7):849-67. doi: 10.1161/CIRCRESAHA.118.311378

92. Pierce GL, Lesniewski LA, Lawson BR, Beske SD, Seals DR. Nuclear factor$\{$ kappa $\} \mathrm{B}$ activation contributes to vascular endothelial dysfunction via oxidative stress in overweight/obese middle-aged and older humans. Circulation (2009) 119(9):1284-92. doi: 10.1161/CIRCULATIONAHA.108.804294

93. Lubos E, Handy DE, Loscalzo J. Role of oxidative stress and nitric oxide in atherothrombosis. Front Biosci (2008) 13:5323-44. doi: 10.2741/3084

94. Ungvari Z, Podlutsky A, Sosnowska D, Tucsek Z, Toth P, Deak F, et al. Ionizing radiation promotes the acquisition of a senescence-associated secretory phenotype and impairs angiogenic capacity in cerebromicrovascular endothelial cells: role of increased DNA damage and decreased DNA repair capacity in microvascular radiosensitivity. J Gerontol A Biol Sci Med Sci (2013) 68(12):1443-57. doi: 10.1093/gerona/glt057 
95. Liu Y, Dong YH, Lyu PY, Chen WH, Li R. Hypertension-Induced Cerebral Small Vessel Disease Leading to Cognitive Impairment. Chin Med J (Engl) (2018) 131(5):615-9. doi: 10.4103/0366-6999.226069

96. Hunziker O, Abdel'Al S, Frey H, Veteau MJ, Meier-Ruge W. Quantitative studies in the cerebral cortex of aging humans. Gerontology (1978) 24(1):2731. doi: $10.1159 / 000212233$

97. Petrenko AG. [Comparative morpho-functional characteristics of the capillary bed of the mammillary bodies and temporal lobe of the human cerebral cortex in young and late middle-aged hypertensive patients]. $\mathrm{Zh}$ Nevropatol Psikhiatr Im S S Korsakova (1987) 87(2):222-5.

98. Attwell D, Mishra A, Hall CN, O'Farrell FM, Dalkara T. What is a pericyte? J Cereb Blood Flow Metab (2016) 36(2):451-5. doi: 10.1177/0271678X15610340

99. Bell RD, Winkler EA, Sagare AP, Singh I, LaRue B, Deane R, et al. Pericytes control key neurovascular functions and neuronal phenotype in the adult brain and during brain aging. Neuron (2010) 68(3):409-27. doi: 10.1016/j.neuron.2010.09.043

100. Armulik A, Genove G, Betsholtz C. Pericytes: developmental, physiological, and pathological perspectives, problems, and promises. Dev Cell (2011) 21 (2):193-215. doi: 10.1016/j.devcel.2011.07.001

101. Toth P, Tucsek Z, Sosnowska D, Gautam T, Mitschelen M, Tarantini S, et al. Age-related autoregulatory dysfunction and cerebromicrovascular injury in mice with angiotensin II-induced hypertension. J Cereb Blood Flow Metab (2013) 33(11):1732-42. doi: 10.1038/jcbfm.2013.143

102. Hall CN, Reynell C, Gesslein B, Hamilton NB, Mishra A, Sutherland BA, et al. Capillary pericytes regulate cerebral blood flow in health and disease. Nature (2014) 508(7494):55-60. doi: 10.1038/nature13165

103. Qin W, Li J, Zhu R, Gao S, Fan J, Xia M, et al. Melatonin protects blood-brain barrier integrity and permeability by inhibiting matrix metalloproteinase- 9 via the NOTCH3/NF-kappaB pathway. Aging (Albany NY) (2019) 11 (23):11391-415. doi: 10.18632/aging.102537

104. Zhang CE, Wong SM, Uiterwijk R, Backes WH, Jansen J, Jeukens C, et al. Blood-brain barrier leakage in relation to white matter hyperintensity volume and cognition in small vessel disease and normal aging. Brain Imaging Behav (2019) 13(2):389-95. doi: 10.1007/s11682-018-9855-7

105. Munoz MS, Chappell FM, Valdes HM, Armitage PA, Makin SD, Heye AK, et al. Integrity of normal-appearing white matter: Influence of age, visible lesion burden and hypertension in patients with small-vessel disease. J Cereb Blood Flow Metab (2017) 37(2):644-56. doi: 10.1177/0271678X16635657

106. Wardlaw JM, Sandercock PA, Dennis MS, Starr J. Is breakdown of the bloodbrain barrier responsible for lacunar stroke, leukoaraiosis, and dementia? Stroke (2003) 34(3):806-12. doi: 10.1161/01.STR.0000058480.77236.B3

107. Henning EC, Warach S, Spatz M. Hypertension-induced vascular remodeling contributes to reduced cerebral perfusion and the development of spontaneous stroke in aged SHRSP rats. J Cereb Blood Flow Metab (2010) 30(4):827-36. doi: 10.1038/jcbfm.2009.246

108. Obermeier B, Daneman R, Ransohoff RM. Development, maintenance and disruption of the blood-brain barrier. Nat Med (2013) 19(12):1584-96. doi: $10.1038 / \mathrm{nm} .3407$

109. Hase Y, Horsburgh K, Ihara M, Kalaria RN. White matter degeneration in vascular and other ageing-related dementias. J Neurochem (2018) 144 (5):617-33. doi: 10.1111/jnc.14271

110. Joutel A, Chabriat H. Pathogenesis of white matter changes in cerebral small vessel diseases: beyond vessel-intrinsic mechanisms. Clin Sci (Lond) (2017) 131(8):635-51. doi: 10.1042/CS20160380

111. Zhang CE, Wong SM, van de Haar HJ, Staals J, Jansen JF, Jeukens CR, et al. Blood-brain barrier leakage is more widespread in patients with cerebral small vessel disease. Neurology (2017) 88(5):426-32. doi: 10.1212/ WNL.0000000000003556
112. Rouhl RP, Damoiseaux JG, Lodder J, Theunissen RO, Knottnerus IL, Staals J, et al. Vascular inflammation in cerebral small vessel disease. Neurobiol Aging (2012) 33(8):1800-6. doi: 10.1016/j.neurobiolaging.2011.04.008

113. Satizabal CL, Zhu YC, Mazoyer B, Dufouil C, Tzourio C. Circulating IL-6 and CRP are associated with MRI findings in the elderly: the 3C-Dijon Study. Neurology (2012) 78(10):720-7. doi: 10.1212/WNL.0b013e318248e50f

114. Shoamanesh A, Preis SR, Beiser AS, Vasan RS, Benjamin EJ, Kase CS, et al. Inflammatory biomarkers, cerebral microbleeds, and small vessel disease: Framingham Heart Study. Neurology (2015) 84(8):825-32. doi: 10.1212/ WNL.0000000000001279

115. Sansoni P, Vescovini R, Fagnoni F, Biasini C, Zanni F, Zanlari L, et al. The immune system in extreme longevity. Exp Gerontol (2008) 43(2):61-5. doi: 10.1016/j.exger.2007.06.008

116. De Martinis M, Franceschi C, Monti D, Ginaldi L. Inflamm-ageing and lifelong antigenic load as major determinants of ageing rate and longevity. FEBS Lett (2005) 579(10):2035-9. doi: 10.1016/j.febslet.2005.02.055

117. Olivieri F, Rippo MR, Procopio AD, Fazioli F. Circulating inflamma-miRs in aging and age-related diseases. Front Genet (2013) 4:121. doi: 10.3389/ fgene.2013.00121

118. Olivieri F, Rippo MR, Monsurro V, Salvioli S, Capri M, Procopio AD, et al. MicroRNAs linking inflamm-aging, cellular senescence and cancer. Ageing Res Rev (2013) 12(4):1056-68. doi: 10.1016/j.arr.2013.05.001

119. Mishra MK, Wang J, Keough MB, Fan Y, Silva C, Sloka S, et al. Laquinimod reduces neuroaxonal injury through inhibiting microglial activation. Ann Clin Transl Neurol (2014) 1(6):409-22. doi: 10.1002/acn3.67

120. Guan D, Li Y, Peng X, Zhao H, Mao Y, Cui Y. Thymoquinone protects against cerebral small vessel disease: Role of antioxidant and antiinflammatory activities. J Biol Regul Homeost Agents (2018) 32(2):225-31.

121. Lampron A, Pimentel-Coelho PM, Rivest S. Migration of bone marrowderived cells into the central nervous system in models of neurodegeneration. J Comp Neurol (2013) 521(17):3863-76. doi: 10.1002/cne.23363

122. Doring A, Sloka S, Lau L, Mishra M, van Minnen J, Zhang X, et al. Stimulation of monocytes, macrophages, and microglia by amphotericin $\mathrm{B}$ and macrophage colony-stimulating factor promotes remyelination. J Neurosci (2015) 35(3):1136-48. doi: 10.1523/JNEUROSCI.1797-14.2015

123. Chen Y, Liu S, Leng SX. Chronic Low-grade Inflammatory Phenotype (CLIP) and Senescent Immune Dysregulation. Clin Ther (2019) 41(3):4009. doi: 10.1016/j.clinthera.2019.02.001

124. Chen Y, Hu M, Gong H. Correlation analysis between the LDL-C in serum and the onset of transient ischemic attack caused by CSVD. Exp Ther Med (2017) 14(2):1119-25. doi: 10.3892/etm.2017.4583

125. Mocchegiani E, Costarelli L, Giacconi R, Cipriano C, Muti E, Tesei S, et al. Nutrient-gene interaction in ageing and successful ageing. A single nutrient (zinc) and some target genes related to inflammatory/immune response. Mech Ageing Dev (2006) 127(6):517-25. doi: 10.1016/j.mad.2006.01.010

Conflict of Interest: The authors declare that the research was conducted in the absence of any commercial or financial relationships that could be construed as a potential conflict of interest.

Copyright () $2020 \mathrm{Jian}, \mathrm{Hu}, \mathrm{Cai}$, Zhang and Lu. This is an open-access article distributed under the terms of the Creative Commons Attribution License (CC BY). The use, distribution or reproduction in other forums is permitted, provided the original author(s) and the copyright owner(s) are credited and that the original publication in this journal is cited, in accordance with accepted academic practice. No use, distribution or reproduction is permitted which does not comply with these terms. 